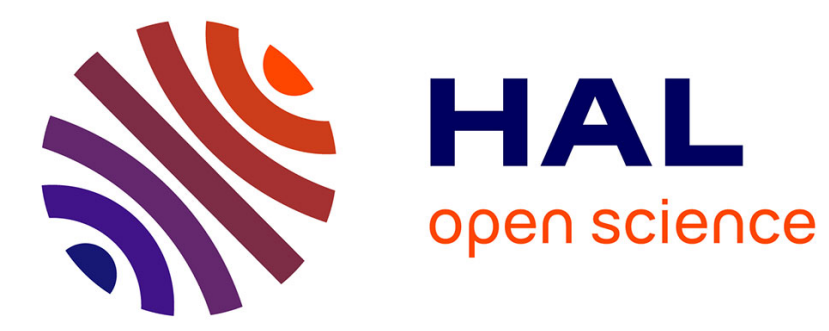

\title{
Description of plasmon-like band in silver clusters: The importance of the long-range Hartree-Fock exchange in time-dependent density-functional theory simulations
}

Franck Rabilloud

\section{- To cite this version:}

Franck Rabilloud. Description of plasmon-like band in silver clusters: The importance of the longrange Hartree-Fock exchange in time-dependent density-functional theory simulations. Journal of Chemical Physics, 2014, 141 (14), pp.144302. 10.1063/1.4897260 . hal-02309832

\section{HAL Id: hal-02309832 \\ https://univ-lyon1.hal.science/hal-02309832}

Submitted on 30 Nov 2020

HAL is a multi-disciplinary open access archive for the deposit and dissemination of scientific research documents, whether they are published or not. The documents may come from teaching and research institutions in France or abroad, or from public or private research centers.
L'archive ouverte pluridisciplinaire HAL, est destinée au dépôt et à la diffusion de documents scientifiques de niveau recherche, publiés ou non, émanant des établissements d'enseignement et de recherche français ou étrangers, des laboratoires publics ou privés. 
Description of plasmon-like band in silver clusters: the importance of the long-range Hartree-Fock exchange in time-dependent density-functional theory simulations

Franck Rabilloud ${ }^{1}$

Institut Lumière Matière, UMR5306 Université Lyon 1 - CNRS,

Université de Lyon 69622 Villeurbanne Cedex, France

(Dated: 3 October 2014)

Absorption spectra of $\mathrm{Ag}_{20}$ and $\mathrm{Ag}_{55}^{q}(q=+1,-3)$ nanoclusters are investigated in the framework of the time-dependent density functional theory (TDDFT) in order to analyse the role of the $d$ electrons in plasmon-like band of silver clusters. The description of the plasmon-like band from calculations using density functionals containing an amount of Hartree-Fock exchange at long range, namely hybrid and range-separated hybrid ( $\mathrm{RSH}$ ) density functionals, is in good agreement with the classical interpretation of the plasmon-like structure as a collective excitation of valence s-electrons. In contrast, using local or semi-local exchange functionals (GGAs or meta-GGAs) leads to a strong overestimation of the role of $d$ electrons in the plasmon-like band. The semi local asymptotically corrected model potentials also describe the plasmon as mainly associated to $d$ electrons, though calculated spectra are in fairly good agreement with those calculated using the RSH scheme. Our analysis shows that a portion of non-local exchange modifies the description of the plasmon-like band. 


\section{INTRODUCTION}

The optical properties of metal nanoparticles is a topic of great fundamental and technological interest. In particular, there has been a great interest in understanding the interaction between light and noble metal nanoparticles for several decades due to their ability to support surface plasmons. Excitations of the plasmon, with a tunable wavelength by adjusting the size or shape of the nanoparticle, leads to a strong absorption band in the UV-visible region ${ }^{1,2}$. Medium-sized and small-sized noble metal clusters have been shown to be also characterized by a strong optical response in the UV-visible range ${ }^{3-21}$. In the case of silver clusters, previous experimental and theoretical works have shown that absorption spectra of very small clusters, $\operatorname{Ag}_{n}(n \leq 12)$, are characterized by several narrow or broad peaks in the $3-5 \mathrm{eV}$ range $\mathrm{e}^{3,4,7,22}$, while for $n \geq 12$ they are characterized by the emergence of a dominant and broad band between 3 and $4 \mathrm{eV}$ and with very strong oscillator strengths ${ }^{8-11,13,14,16,17,20,23}$. The transition from molecular-like, characterized by several well-separated peaks, to plasmon-like spectra characterized by a single and broad band in the UV-visible domain occurs between $n=12$ and $n=20^{13}$.

Theoretically, the description of the optical response of noble metal clusters remains an interesting challenge due to the close proximity of the $d$ electrons which affect spectra by quenching the oscillator strengths through screening of the $s$ electrons, i.e. the $d$ electrons form a polarizable background that strongly screens the valence $s$ electron interactions, and by being involved in the excitation. The observed spectra for silver clusters were first interpreted successfully using classical electrodynamics by solving Maxwell's equations for electromagnetic waves interacting with small spherical metallic particles (Mie theory), or ellipsoidal particles (Mie-Gans theory), characterized by the dielectric function of the bulk ${ }^{11,14}$. Some more sophisticated semiclassical theories, using for examples the RPA (Random Phase Approximation) description ${ }^{24}$ or approaches based on time-dependent local density approximation and jellium models ${ }^{25-27}$, have been developed to better describe the core polarization and its effect on the optical response of silver clusters. In all these classical or semiclassical theories, the plasmon resonance reflects a collective excitation of the $s$ valence electrons, while the effects of the $d$-electrons are only accounted for by using the bulk dielectric function.

More recently, a few studies have been performed within a fully quantum treatment for all 
electrons in the framework of the time-dependent density functional theory ${ }^{28-30}$ (TDDFT) on silver clusters of some tens of atoms ${ }^{4,13-15,18,31-33}$. Calculated absorption spectra were in good agreement with the experimental ones, in particular the plasmon-like structure of $\mathrm{Ag}_{18}, \mathrm{Ag}_{20}, \mathrm{Ag}_{22}, \mathrm{Ag}_{55}, \mathrm{Ag}_{140}$ were well reproduced. However, the analyses of the electronic excitations, based either on the plot of the electron density involved in the excitations ${ }^{4,13}$ or on approximate calculations of the percentage of the $d$ character in the transitions ${ }^{14,31}$, concluded that the $d \rightarrow s p$ interband transitions have a significant contribution (at least 40\%) to optical excitations. For example, in our previous study ${ }^{13}$ the transitions associated to the plasmon were found to be due to excitations from inner orbitals, with a shape associated to $d$ contributions, to outer region with an $s+p$ character. Thus, quantum chemistry calculations were found to give a description of the absorption spectra in which the role of $d$ electrons is important. That contrasted with semi-classical theories commonly used to explain spectra of silver nanoparticles, in which the transitions are described by a collective excitation of $s$ valence electrons while the effects of $d$ electrons are just taken into account through a phenomenological dielectric function. In quantum calculations, the $d$-type electrons were found to be more active, and the $d \rightarrow s p$ interband transitions to be more important.

The goal of the present paper is to understand the apparent disagreement between the semiclassical theories and previous TDDFT calculations. Let us note that both approaches give somewhat similar absorption spectra, in fairly good agreement with the experimental data, while they disagree on the description of the excitations. Previous TDDFT calculations ${ }^{4,13-15,18,31}$ were restricted to the adiabatic linear-response formulation using local or semi local density approximations, i.e. either LDA (local-density approximation) or standard GGA (generalized gradient approximations) density functionals. But it is well known that the exchange-correlation potential derived from these conventional functionals, $v_{x c}(r)=\delta E_{x c}[\rho] / \delta \rho(r)$, have an incorrect asymptotic form which leads to errors in optical properties. Indeed, the potential of pure density functionals decays to zero exponentially rather than as $-1 / r$ at large $r$. As a consequence it is less attractive at long range than the exact potential. This may result into a poor description of higher occupied orbitals and virtual orbitals, and then a poor performance for calculating excitation energies. Beside an accurate continuous potential should not vanish asymptotically, but should tend to a positive constant $\Delta_{\infty}$ as a consequence of the integer derivative discontinuity in the exchange-correlation energy ${ }^{34,35}$. Tozer and coworkers ${ }^{35}$ have suggested to use the limit 
$\Delta_{\infty}=I+\epsilon_{\text {HOMO }}$, where I and $\epsilon_{\text {HOMO }}$ are the lowest ionization energy and the orbital energy of the highest occupied molecular orbital respectively. Various asymptotically corrected potentials have been constructed. For examples, $\mathrm{LB}^{36}$ and $\mathrm{SAOP}^{37}$ are local potentials which give the correct $-1 / r$ behavior, but they vanish at infinity. BP-GRAC-LB ${ }^{38}$, based on the LB94 potential, gives a more correct long-range asymptote as $-1 / r+I+\epsilon_{\text {HOMO }}$. It is also well known that TDDFT using current local or semi-local functionals has problems with the calculation of charge-transfer excited states ${ }^{39}$. The excitations are much low and the potential energy curves do not exhibit the correct asymptote in $1 / R$ where $R$ is the distance between the positive and the negative charges of the charge-transfer state. This failure has been understood as an electron-transfer self-interaction error ${ }^{40}$. To give a more suitable description of charge-transfer excitations in the adiabatic linear-response approximation, a long-range correction should be applied to the exchange functional. In the range-separated hybrid ( $\mathrm{RSH}$ ) functionals ${ }^{41}$, also known as long-range corrected (LC) functionals, the Coulomb operator is then split into two parts:

$$
\frac{1}{r_{12}}=\frac{1-\operatorname{erf}\left(\omega r_{12}\right)}{r_{12}}+\frac{\operatorname{erf}\left(\omega r_{12}\right)}{r_{12}},
$$

where $r_{12}$ is the distance between two electrons, and $\omega$ is a range separation parameter. The first term of the right-hand side is a short-range term evaluated with the exchange potential from DFT, while the second term, the long-range part, is evaluated with the nonlocal Hartree-Fock (HF) exchange. The $\omega$ value has been optimized to use the above partition in combination with various standard density functionals. The exchange at shortrange can also be evaluated with a hybrid functional, i.e. containing a portion of $\mathrm{HF}$ exchange, and in that case the resulting LC functional is a global hybrid with an amount of HF exchange at both short and long ranges. The scheme of equation (1) resolves a significant part of the self-interaction-error (SIE) problems, and also improve the asymptotic behavior at long range. The accuracy of several long-range corrected density functionals for calculating the vertical absorption spectra of silver clusters in the adiabatic linear-response formulation of TDDFT has been recently evaluated ${ }^{23}$.

We present here new TDDFT calculations performed with several types of functionals (GGA, meta-GGA, hybrid, and RSH functionals) and asymptotically corrected model potentials (LB94, SAOP, BP-GRAB-LB). New analysis will allow us to discuss the validity of the description of the plasmon-like band within the TDDFT formalism. We will show that 
the use of Hartree-Fock exchange at long range leads to results in fairly good agreement with the classical interpretation of plasmon-like structure as a collective excitation of valence $s$ electrons, contrarily to the use of GGA-type density functionals that leads to overestimate the role of $d$ electrons in excitations. The local or semi local asymptotically corrected models may give correct spectra but they also tend to overestimate the contribution of $d$ electrons. We will present absorption spectra of $\operatorname{Ag}_{n}$ with $n=20$ and 55. In the case of $\mathrm{Ag}_{55}$, we will only consider the charged species, namely $\mathrm{Ag}_{55}^{+}$and $\mathrm{Ag}_{55}^{3-}$, to reduce the computational cost. As the effects of spin orbit coupling were shown to be not significant in silver clusters ${ }^{42}$, only scalar relativistic corrections have been considered in the present study.

\section{COMPUTATIONAL DETAILS}

Calculations were performed using both non-asymptotically corrected exchange-correlation functionals (GGA, meta-GGA, and hybrid functionals) and two families of long-rangecorrected density functionals methods (non-local LC functionals and semi local asymptotically corrected model potentials). We briefly give below some details of calculations.

The following exchange-correlation functionals of GGA type were considered: $\mathrm{BP} 86^{43,44}$, $\mathrm{PBE}^{45}, \mathrm{BLYP}^{43,46}$, PW91 47 . Two meta-GGA functionals ("meta" denotes the inclusion of kinetic energy density, which depends on local derivatives of the spin orbitals) were tested: M06L which was parametrized to have good performances for transition metal chemistry and to satisfy the uniform-electron-gas limit ${ }^{48}$ and TPSS, a non-empirical functional designed for molecules and solids ${ }^{49}$. The LC-functionals LC-BP86 $(\omega=0.47)^{50}$ and LC-M06L $(\omega=$ $0.15,0.33,0.47,0.60,0.80)$ which contain $0 \%$ Hartree-Fock exchange at short range and $100 \%$ at long range were considered. The popular hybrid functional B3LYP ${ }^{51}$ and the hybrid metaGGA M06-2X ${ }^{52}$ were also used. The long-range-corrected global hybrid density functionals, CAM-B3LYP 53 and $\omega$ B97 ${ }^{54}$, were also considered. The first one, which combines the hybrid B3LYP functional at short range with an increasing amount of exact Hartree-Fock exchange at long-range, comprises of 19\% Hartree-Fock exchange at short-range and $65 \%$ HartreeFock at long-range. In the case of $\omega \mathrm{B} 97 \mathrm{x}$, the percentage of Hartree-Fock exchange is $16 \%$ at short-range and $100 \%$ at long-range. Finally, we also used LC- $\omega \operatorname{PBE}(\omega=0.20,0.40,0.60)^{55}$. Calculations were performed with the Gaussian09 suite of programs ${ }^{56}$. Silver atoms were described through a relativistic effective core potential (RECP) so that only 19 valence 
electrons were treated explicitly ${ }^{57,58}$. The corresponding Gaussian basis set was 8s7p6d contracted into 6s5p3d ${ }^{57}$ in the case of $\mathrm{Ag}_{20}$, and $7 \mathrm{~s} 6 \mathrm{p} 4 \mathrm{~d}$ contracted into $3 \mathrm{~s} 3 \mathrm{p} 2 \mathrm{~d}^{58}$ for $\mathrm{Ag}_{55}^{+}$. For calculations on $\mathrm{Ag}_{55}^{3-}$, some $s$ - and $p$-type diffuse functions were added. We give in Supplemental materials ${ }^{59}$ some spectra calculated using several RECP R7,58,60 $^{5}$ and basis sets of double, triple and quadruple zeta valence + polarization quality ${ }^{61,62}$. They show that the absorption spectra are reproducible whatever the basis set and RECP used.

We have also performed some calculations with several asymptotically corrected semi local model potentials, namely LB94, SAOP and BP-GRAC-LB, using the program ADF ${ }^{63}$. Scalar relativistic effects were included with the zeroth-order regular approximation (ZORA) to the Dirac equation $^{64,65}$. We have used double- $\zeta$ (DZ), triple- $\zeta$ plus polarization (TZP or TZ2P), and quadruple- $\zeta$ plus polarization (QZ4P) Slater-type basis sets. For calculations with LB94 and BP-GRAC-LB models, silver atoms were represented through an effective core pseudopotential. In the small core pseudopotential (Ag.3d) version, silver was considered as $\mathrm{Ag}^{19+}$ core (inner core orbitals have been kept frozen up to the $3 \mathrm{~d}$ shell) with $4 s, 4 p, 4 d$ and $5 s$ active electrons. In the large core pseudopotential (Ag.4p) version, only $4 d$ and $5 s$ electrons were treated explicitly. For the BP-GRAC-LB calculations, the ionization potential was derived from BP86 calculations on the neutral and cationic species.

As discussed in the introduction, we can distinguish the long-range-corrected density functional methods into two families : the LC hybrid, or RSH, functionals and the semi local asymptotically corrected (AC) model potentials. For the LC hybrid functionals, some fraction of the non local Hartree-Fock ( $\mathrm{HF}$ ) exchange for the long-range interactions is added to a conventional functional (GGA, meta-GGA, or hybrid) following the equation (1). LCBP86,LC- $\omega$ PBE, LC-M06L, CAM-B3LYP, $\omega$ B97x are in this family. In contrast, in the AC model potential scheme, the exchange-correlation potential is modeled at long range and added to a GGA functional. In this family we can find LB94, BP-GRAC-LB, SAOP.

We have considered the following clusters: $\mathrm{Ag}_{20}, \mathrm{Ag}_{55}^{+}$and $\mathrm{Ag}_{55}^{3-}$. Most calculations have been performed on $\mathrm{Ag}_{20}$ because its absorption spectrum presents a plasmon-like band and its size is small enough to do intensive calculations and analysis. The geometry of $\mathrm{Ag}_{20}$ is the lowest-energy structure obtained at BP86 level in a previous work ${ }^{13}$. It was locally optimized with each functional before doing the present calculation of optical properties. The structure of $\mathrm{Ag}_{55}^{+}$and $A g_{55}^{3-}$ is the $I_{h}$ symmetry structure optimized at BP86 level. The geometrical structure of clusters are shown in Supplemental materials ${ }^{59}$. 

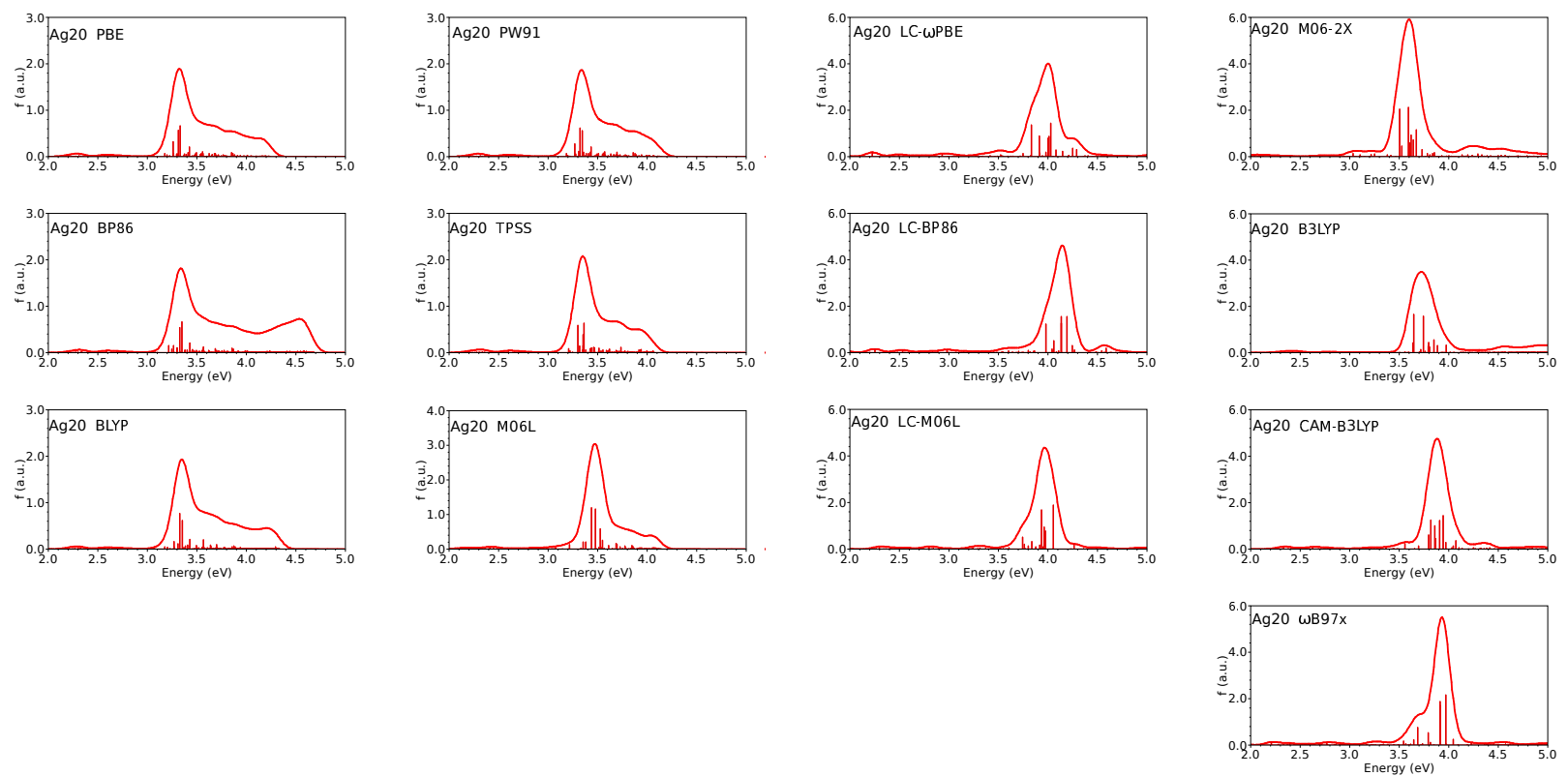

FIG. 1. Absorption spectra of $\mathrm{Ag}_{20}$. The value of $\omega$ is 0.40 and 0.33 in LC- $\omega \mathrm{PBE}$ and LC-M06L respectively.

The absorption spectra showed in the next section give the oscillator strength as a function of the excitation energy together with a curve obtained by a Gaussian broadening with a full width at half maximum (fwhm) of $0.08 \mathrm{eV}$. Pre- and post-processing operations were performed by using the graphical interface Gabedit ${ }^{66}$.

\section{RESULTS AND DISCUSSION}

\section{A. Absorption spectra of $\mathbf{A g}_{20}$}

Calculated spectra of $\mathrm{Ag}_{20}$ obtained with several density functionals are shown in Figure 1. Spectra were calculated in the 2.0-5.0 eV range except when GGA and meta-GGA density functionals were used because of the high density of electronic states with many spurious states $^{23}$ and the unreasonable computational cost. All calculated spectra are characterized by a dominant and relatively broad band between 3 and $4.5 \mathrm{eV}$. The feature is similar to the plasmon excitation observed in metal nanoparticles of several $\mathrm{nm}$ in diameter. To our knowledge, no experimental spectrum of $\mathrm{Ag}_{20}$ in gas phase is available. However, the spectrum of $\mathrm{Ag}_{20}$ has been measured on clusters embedded in solid argon ${ }^{11}$. It shows a band centered at $3.73 \mathrm{eV}$ with a width of about $0.45 \mathrm{eV}$. Based on the classical Mie approach, 
Fedrigo et al. ${ }^{11}$ have estimated that the plasmon-like band in silver clusters was likely to be redshifted by $0.24 \mathrm{eV}$ when the clusters are embedded in argon matrix with respect to the gas phase. Considering the dipolar plasmon resonance of large particules, Haberland ${ }^{67}$ estimated this redshift at $0.29 \mathrm{eV}$. Otherwise, the effects of the argon matrix were estimated using an electrostatic model of solvation (the well know conductor-like screening model of solvation (COSMO) model) in TDDFT/GGA calculations ${ }^{68}$, the redshift was calculated to be $0.17 \mathrm{eV}$. Therefore, we can reasonably assume that in gas phase the plasmon-like band of $\mathrm{Ag}_{20}$ is centered in the range 3.9-4.1 eV.

Using GGA or meta-GGA type density functionals, namely PBE, PW91, BP86, BLYP, TPSS, and M06L, the calculated band is centered at about 3.2-3.3 eV, while the use of longrange corrected functionals leads to a blueshift of $0.5-0.8 \mathrm{eV}$ and then spectra compare well with the experimental one. Beside one can note others major differences between spectra calculated at GGA or meta-GGA levels and those obtained using hybrid functionals. The main band calculated at GGA or meta-GGA levels is composed of two or three main peaks surrounded by many transitions with weak oscillator strengths and well scattered resulting in a very large structure. The spectra are biased by many spurious states beyond $3 \mathrm{eV}$. The GGA and meta-GGA spectra do not reproduce so well the plasmon-like band of the experimental spectrum because a strong bottom signal still exists beyond the main band while no transition is measured in experiments. In contrast, spectra calculated with LC functionals, including LC-GGA, LC-metaGGA and LC global hybrid functionals (namely LC-BP86, LC-M06L, LC- $\omega$ PBE, CAM-B3LYP, $\omega$ B97x respectively), present a main band originated from five main peaks with strong oscillator strengths, and then very few transitions beyond the plasmon band. The density of states are much lower than that obtained with GGA density functionals. Moreover, at GGA and meta-GGA levels the main transitions are due to the excitations from orbitals with a shape associated to $s$ and $d$ contributions, to the outer region with an $s+p$ character distributed on the whole cluster. Important contributions come from inner $d$-orbitals HOMO- $n, n=6-21$. In contrast, the role of $d$-electrons is less important in calculations with LC-functionals since the transitions are mainly due to excitations from HOMO- $n(n=0-5) s$-type orbitals. The excitations from $d$-orbitals are pushed to much higher energies (beyond $5 \mathrm{eV}$ ).

In order to quantify the respective contributions of $s$ and $d$ electrons to the optical response, we have calculated the percentage of the $s, p$ and $d$ characters in the transition 
thanks to the formula proposed by Baisha ${ }^{14}$. For the contribution of $d$ electrons, it is read as followed:

$$
\% d=\frac{\sum_{n} f_{n} \sum_{v c}\left|F_{n}^{v c}\right|^{2}\left|\left\langle d \mid \phi_{v}\right\rangle\right|^{2}}{\sum_{n} f_{n}} \times 100
$$

where the sum in $n$ includes all the excitations that form the plasmon band. For one transition with an oscillator strength $f_{n}$, the double index $v c$ labels the entries of the corresponding TDDFT eigenvector $F_{n}$, which is composed of occupied-unoccupied (or "valenceconduction") Kohn-Sham orbital pairs. Of course $\sum_{v c}\left|F_{n}^{v c}\right|^{2}=1$ for each transition $n$. $\left\langle d \mid \phi_{v}\right\rangle$ is the $d$ projection of the occupied orbital $\phi_{v}$. We have similar equations for $s$ and $p$ contributions. Table I gives the calculated values for several functionals. The percentage of the $d$ character is calculated to be about $40 \%$ at GGA and meta-GGA levels with the exception of M06L for which the percentage is $15.5 \%$. In contrast, the role of $d$ electrons is much less important when a long-range corrected density functional is used. With hybrid CAM-B3LYP and $\omega \mathrm{B} 97 \mathrm{x}$ the percentage of $d$ character is 7.3 and $8.8 \%$ respectively, while it is about $10 \%$ with LC-BP86, LC- $\omega$ PBE, and LC-M06L. The inclusion of the Hartree-Fock exchange at long range pushes the $d \rightarrow s p$ interband transitions to higher energies. For LC- $\omega \mathrm{PBE}$ and LC-M06L, we give results obtained using several values of $\omega$ in the $0.15-0.60$ range. The percentage of $d$ character slightly decreases with increasing $\omega$ value. The percentage of $s$ and $p$ characters are respectively $\sim 35$ and $\sim 25 \%$ with both GGAs and meta-GGAs while they are respectively in the 50-60 and 30-40\% ranges with both hybrid and LC functionals. The $p$ contributions are due to the $s p$ hybridization.

Interestingly, the hybrid B3LYP and M06-2X functionals which contain respectively $20 \%$ and $54 \%$ Hartree-Fock exchange furnish results in good agreement with those obtained with LC functionals even though they are less efficient in removing the spurious states. Indeed the contribution of $d$ electrons are calculated to be 7.0 and $8.4 \%$ with B3LYP and M06-2X respectively (see Table I), while spectra are found to be fairly similar to those obtained with LC functionals. Thus, a relatively small amount of Hartree-Fock exchange at long-range is sufficient to remove the main spurious interband transitions.

As results based on the equation (2) are expected to depend on the basis set, we have tested other basis sets, namely QZVP and LanL2DZ, with the LC-M06L( $\omega=0.47)$ functional. The absorption spectra, given in Supplemental materials ${ }^{59}$, are very similar to those obtained with the SDD basis set (and showed in Figure 1). The percentage of $d$ character are found to be $4.9 \%$ and $2.4 \%$ with QZVP and LanL2Dz basis sets respectively. Those 
TABLE I. Percentage of $s, p, d$ characters in the plasmon band of $\mathrm{Ag}_{20}$ and $\mathrm{Ag}_{55}^{+}$.

\begin{tabular}{|c|c|c|c|}
\hline $\mathrm{Ag}_{20}$ & $\mathrm{~s}$ & $\mathrm{p}$ & $\mathrm{d}$ \\
\hline BP86 & 34.9 & 25.4 & 39.7 \\
\hline BLYP & 37.0 & 24.8 & 38.2 \\
\hline PBE & 35.0 & 26.3 & 38.7 \\
\hline PW91 & 35.6 & 25.6 & 38.8 \\
\hline TPSS & 32.4 & 25.4 & 42.2 \\
\hline M06L & 57.0 & 27.5 & 15.5 \\
\hline M06-2X & 62.6 & 29.0 & 8.4 \\
\hline B3LYP & 57.3 & 35.7 & 7.0 \\
\hline $\operatorname{CAM}-B 3 L Y P(\omega=0.33)$ & 58.6 & 34.1 & 7.3 \\
\hline LC-BP86 $(\omega=0.47)$ & 57.2 & 31.9 & 10.8 \\
\hline $\operatorname{LC}-\omega \operatorname{PBE}(\omega=0.20)$ & 47.6 & 41.3 & 11.1 \\
\hline $\operatorname{LC}-\omega \operatorname{PBE}(\omega=0.40)$ & 58.5 & 29.5 & 12.0 \\
\hline $\operatorname{LC}-\omega \operatorname{PBE}(\omega=0.60)$ & 65.5 & 24.9 & 9.6 \\
\hline $\mathrm{LC}-\mathrm{M} 06 \mathrm{~L}(\omega=0.15)$ & 51.1 & 38.1 & 10.8 \\
\hline $\mathrm{LC}-\mathrm{M} 06 \mathrm{~L}(\omega=0.33)$ & 53.9 & 37.1 & 9.0 \\
\hline $\mathrm{LC}-\mathrm{M} 06 \mathrm{~L}(\omega=0.47)$ & 55.6 & 35.1 & 9.3 \\
\hline $\mathrm{LC}-\mathrm{M} 06 \mathrm{~L}(\omega=0.60)$ & 57.1 & 35.1 & 7.8 \\
\hline$\omega \operatorname{Bg} 97 x(\omega=0.30)$ & 56.2 & 35.0 & 8.8 \\
\hline $\mathrm{Ag}_{55}^{+}$ & $\mathrm{s}$ & $\mathrm{p}$ & d \\
\hline $\mathrm{LC}-\mathrm{M} 06 \mathrm{~L}(\omega=0.33)$ & 25.4 & 70.7 & 3.9 \\
\hline $\mathrm{LC}-\mathrm{M} 06 \mathrm{~L}(\omega=0.47)$ & 23.2 & 74.5 & 2.3 \\
\hline $\operatorname{LC}-\mathrm{M} 06 \mathrm{~L}(\omega=0.8)$ & 23.0 & 75.7 & 1.3 \\
\hline $\operatorname{CAM}-B 3 L Y P(\omega=0.33)$ & 31.1 & 61.5 & 7.4 \\
\hline
\end{tabular}

values confirm the relatively small role of the $d \rightarrow s p$ interband transitions in the plasmon.

To further characterize the excitations in our TDDFT calculations, we show in Figure 2 a plot of the electron density difference between the excited and the ground states for some representative main peaks. Red colored regions correspond to depletion of the electron 
density during the transition, while blue regions correspond to an increase of the electron density. More isosurfaces are given in Supplemental materials ${ }^{59}$. Plots show clearly that, in the case of GGA density functionals (BP86 and PBE), transitions are due to the excitations from inner orbitals, with a shape associated to $d$ and $s$ contributions, to outer region distributed on the whole cluster and with an $s+p$ character. In particular, the densities of $d$-type orbitals located on silver atoms are clearly visible. On the contrary, using hybrid functionals, the transitions are associated to $s$ and $p$ orbitals with very few $d$ contributions. In all cases, the accumulation of the electrons is found to be done outside the cluster. Those plots confirm that the role of $d$ electrons appears to be less important when using non local exchange.

The inclusion of an amount of Hartree-Fock exchange at long range in corrected functionals is crucial to describe excitations of Rydberg and charge-transfer character for which the spatial overlap between the occupied and virtual orbitals is small. In these cases, a significant underestimation of TDDFT excitation energies is expected when using GGA-type density functionals. In the present work, we have evaluated the spatial overlap in a given excitation using the $\Lambda$ diagnostic test proposed by Tozer ${ }^{39}$ :

$$
\Lambda=\frac{\sum_{v c}\left|F^{v c}\right|^{2} O_{v c}}{\sum_{v c}\left|F^{v c}\right|^{2}},
$$

where the sum includes all occupied-virtual (or "valence-conduction") pairs which contribute to the excitation, $F^{v c}$ is similar to $F_{n}^{v c}$ in equation (2), and $O_{v c}$ is the product of the moduli of the two orbitals defined as followed

$$
O_{v c}=\left\langle\left|\phi_{v}\right||| \phi_{c} \mid\right\rangle=\int\left|\phi_{v}(\vec{r})\right|\left|\phi_{c}(\vec{r})\right| d \vec{r}
$$

$\Lambda$ takes values in the interval $[0,1]$. Following previous tests on 18 molecules $^{39}$, the value of $\Lambda$ can be helpful to distinguish the three categories of excitations: the local excitations $(\Lambda \geq 0.45)$, the Rydberg excitations $(0.08 \leq \Lambda \leq 0.27)$, and the charge-transfer excitations $(0.06 \leq \Lambda \leq 0.72)$. Note that the latter cover a wide range of overlaps. For the main transitions involved in the plasmon-like band, we found a value of $\Lambda \sim 0.6$ whatever the density functional used, the calculated values being in the 0.53-0.61 range with GGA-type functionals and in the 0.60-0.69 range using LC functionals. In spectra calculated with GGA-type density functionals, the excitations in the 3.5-4.5 eV range and located beyond the plasmon band are due to transitions from purely $d$-type orbitals to outer region with 
BP86 (3.35 eV)
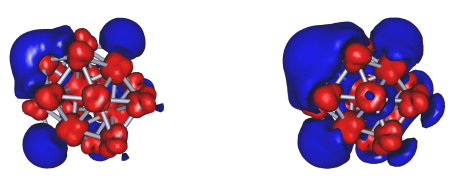

PBE (3.33 eV)
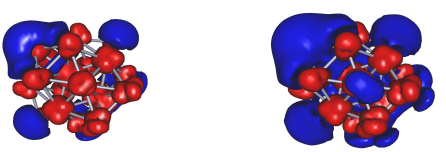

LC-BP86 (4.19 eV)
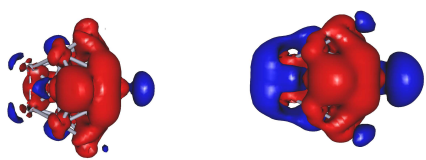

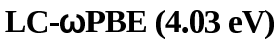
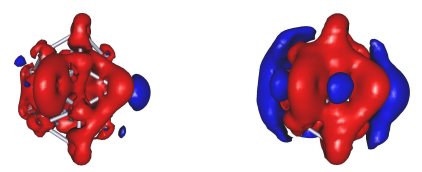

LC-M06L(4.09 eV)
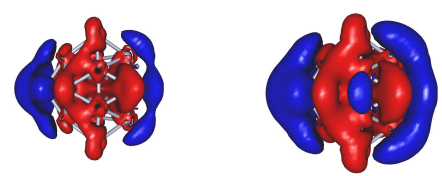

$\omega B 97 x(3.91 \mathrm{eV})$
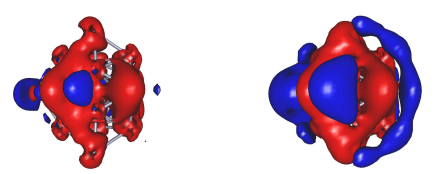

B3LYP (3.74 eV)
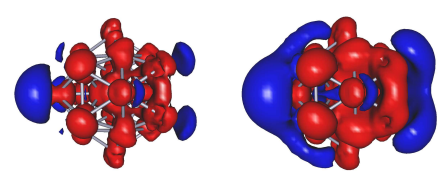

M06-2X (3.60 eV)
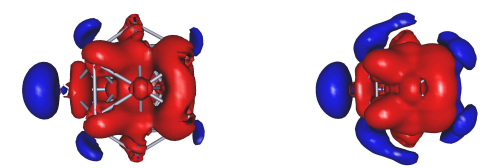

FIG. 2. Isosurface of the electron density difference between the excited and ground states for some of the main peaks of $\mathrm{Ag}_{20}$ clusters. The isovalues are 0.0002 and 0.0001 a.u. respectively. Red colored regions correspond to the depletion of the electron density during the transition while the blue regions correspond to the accumulation of electrons. Additional isosurfaces are given in Supplemental materials ${ }^{59}$.

$s+p$ character. For these transitions, the $\Lambda$ value is calculated to be about 0.40 with PBE, BP86, BLYP, PW91. At GGA level the plasmon is made of both contributions from $d \rightarrow s p$ interband transitions (with $\Lambda \sim 0.4$ ) and contributions from $s \rightarrow s p$ intraband 
transitions (with $\Lambda \sim 0.7$ ) resulting into a band with a value of $\Lambda$ in the $0.53-0.61$ range. The $d \rightarrow s p$ excitation energies are significantly underestimated at TDDFT/GGA level and consequently the $d \rightarrow s p$ transitions are found in the $3-4 \mathrm{eV}$ range together with the $s \rightarrow s p$ transitions. They are therefore found in the plasmon band. Using LC functionals the $4 d \rightarrow 5 s 5 p$ transitions are pushed beyond about $5 \mathrm{eV}$. Tozer and coworkers have shown that a PBE excitation with $\Lambda<0.4$ is likely to be in very significant error ${ }^{39}$. In the case of $\mathrm{Ag}_{20}$, the value of 0.4 for the $4 d \rightarrow 5 s 5 p$ transitions is on the border line of the Tozer analysis, and it is not easy to attribute the deterioration to the lack of long-range HF exchange correction or to the wrong asymptotic behavior. However, we can conclude that the TDDFT/GGA excitations calculated beyond the plasmon are states which are likely to be found at higher energies.

We show in Figure 3 the spectra calculated using several AC model potentials. LB94 and SAOP give spectra somewhat similar to those calculated with LC hybrid functionals. The plasmon-like band is centered at $3.77 \mathrm{eV}$ at SAOP/QZ4P level. Using LB94, the position of the main band is sensitive to the core pseudopotential since it is centered at 3.92 and 4.10 $\mathrm{eV}$ at Ag.3d/TZ2P and Ag.4p/TZ2P levels respectively. Beside using a smaller basis set, like DZ or TZP, leads to a blueshift of 0.1-0.2 eV (see figures in Supplemental materials ${ }^{59}$ ). In all cases, the transitions are mainly associated to excitation of $s$ electrons with a very few contribution of $d$ electrons. The percentage of the $d$ character in the plasmon band estimated with the equation (2) using the Slater-type basis set is about 10\% with both LB94 and SAOP model potentials. The contributions of $d$ electrons become significant for excitations located above 4.6 and $4.3 \mathrm{eV}$ at LB94 and SAOP levels respectively. Surprisingly, the BP-GRAC-LB model potential gives no improvement with respect to BP86 since the plasmon is found to be at 3.3 and $3.5 \mathrm{eV}$ using the core Ag.3d and Ag.4p respectively. However, the good quality of spectra obtained at both LB94 and SAOP model potential means that the asymptotic behavior as $-1 / r$ at long range is prominent to give a correct description of plasmon-like band of $\mathrm{Ag}_{20}$. Therefore, the use of RSH functionals with the HF exchange at long range gives good results because the HF exchange restores the correct asymptote. Our observation in term of $\Lambda$ and the difference between the RSH and GGA functionals, together with the behavior with regard to the asymptotically corrected functionals, suggests the $4 d \rightarrow 5 s 5 p$ transitions has some Rydberg character. Let us note that LB94 and SAOP calculate about twice excited states than LC hybrid functional do, and so they are less effective in reducing 

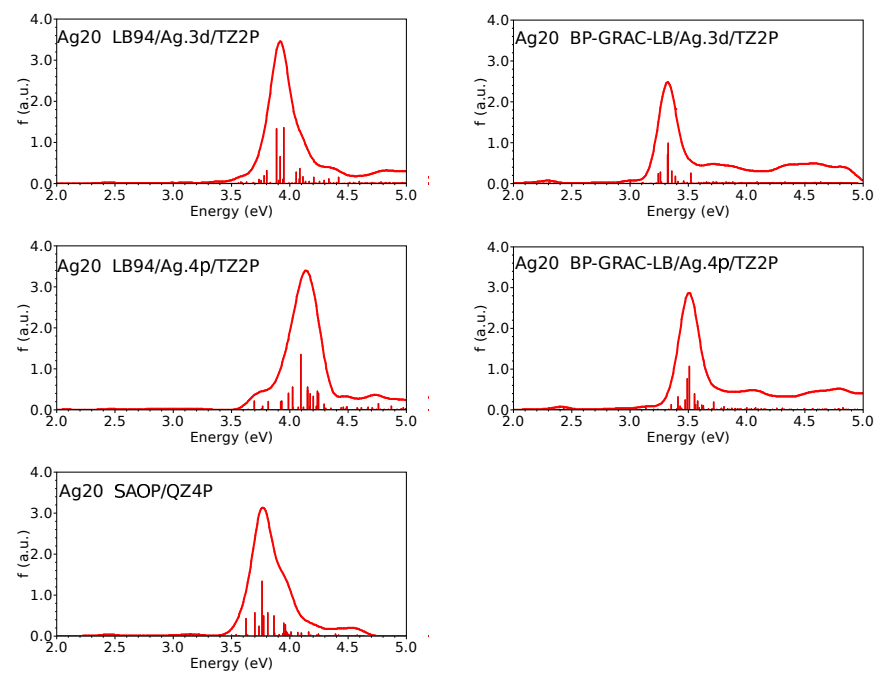

FIG. 3. Absorption spectra of $\mathrm{Ag}_{20}$ using LB94, BP-GRAC-LB and SAOP model potentials.

the occurrence of spurious states.

Finally, we have also calculated the spectrum of $\mathrm{Ag}_{20}$ for the tetrahedral structure as considered previously by Aikens ${ }^{15}$. Although this structure does not compete for the lowestenergy isomer, it is of interest because it corresponds to a shell closing. Let us note that the position of the band is strongly dependent on the basis set and pseudopotential used since it is redshifted by about $0.4 \mathrm{eV}$ when we improve the quality of the basis set from $\mathrm{DZ}$ to QZ4P ${ }^{15}$. For a given basis set, the spectrum of the $\mathrm{T}_{d}$ isomer is somewhat similar to that of the lowest-energy isomer discussed above with however a redshift of about $0.1-0.2 \mathrm{eV}$, for example the plasmon band is located at $3.63 \mathrm{eV}$ for the $\mathrm{T}_{d}$ structure at SAOP/QZ4P level, to be compared to the value of $3.77 \mathrm{eV}$ in Figure 3. The major difference concerns the width of the band, since a single transition is associated to the band in the $\mathrm{T}_{d}$ structure, while a clump of lines is obtained when the symmetry is lowered. Some spectra in the tetrahedral structure can be seen in Supplemental materials ${ }^{59}$.

\section{B. Absorption spectra of $\mathbf{A g}_{55}^{+}$}

Recently, Weissker and coworkers ${ }^{18}$ computed the spectrum of the neutral $\mathrm{Ag}_{55}$ cluster at Real-Time TDDFT level using a GGA functional. They found a main band at about $3.7 \mathrm{eV}$, and a less intense one at about $3.4 \mathrm{eV}$. Calculations by Aikens ${ }^{15}$ at TDDFT/BP86 level on tetrahedral $\mathrm{Ag}_{56}^{+}$cluster show a band centered at about $3.1 \mathrm{eV}$. To our knowledge, 

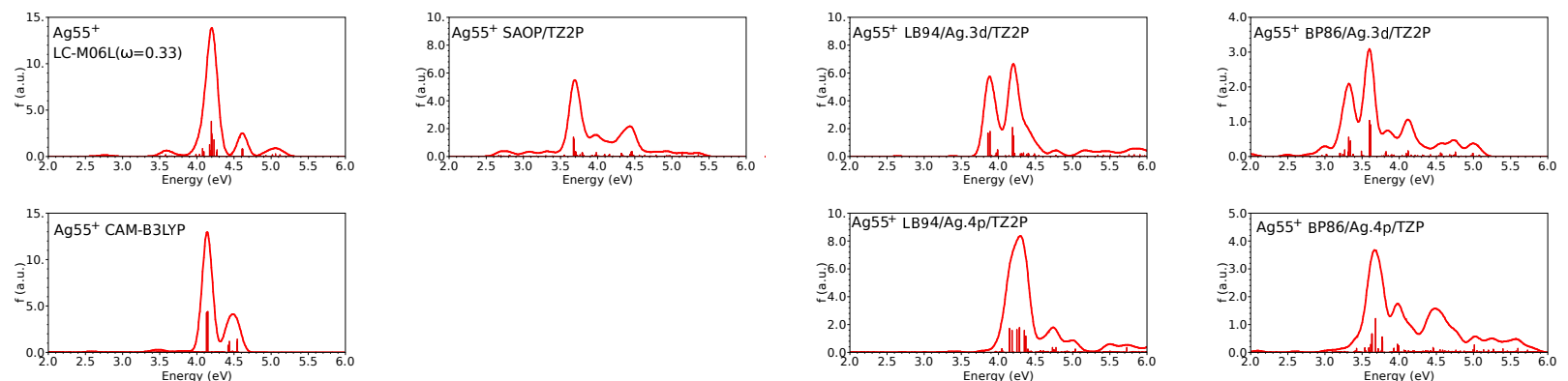

FIG. 4. Absorption spectra of $\mathrm{Ag}_{55}^{+}$.

there is no experimental spectrum on neutral or cationic $\mathrm{Ag}_{55}^{(+)}$cluster, but experiments on clusters of similar sizes $(n=39-60)$ suggest that the spectrum of $\operatorname{Ag}_{55}^{+}$could present a plasmon band in the $3.5-4.5 \mathrm{eV}$ range ${ }^{10,11,69}$.

We show our calculated spectra concerning the $I_{h}$-symmetry $\mathrm{Ag}_{55}^{+}$cluster in Figure 4. Additional spectra with several basis sets and density functionals can be found in Supplemental materials ${ }^{59}$. Calculations using hybrid density functionals, namely B3LYP and M06-2X, have not been considered because of the unreasonable computational cost due to the HF exchange and the very large number of excited states to be calculated. Calculations using a RSH functional is easier because the number of excited states is smaller. The plasmon band is centered at $4.14 \mathrm{eV}$ with the global hybrid CAM-B3LYP functional, and at $4.20 \mathrm{eV}$ with LC-M06L $(\omega=0.33)$. It is blueshifted at 4.30 and $4.40 \mathrm{eV}$ when using LC-M06L with $\omega=0.47,0.80$ respectively. However, we have shown in a previous work ${ }^{23}$ that $\omega=0.33$ is the best choose to reproduce experimental data on small $\mathrm{Ag}_{n}$ clusters, hence we consider the value of $4.20 \mathrm{eV}$ as the best prediction obtained at LC-M06L level. For both CAM-B3LYP and LC-M06L, a less-intense peak is found at about $0.35 \mathrm{eV}$ beyond the plasmon band. The spectrum calculated using the potential model SAOP shows a main band centered at 3.68 $\mathrm{eV}$ followed by a two less-intense peaks at 4 and $4.5 \mathrm{eV}$. Contrary to spectra obtained with the above LC functionals in which only few transitions with giant oscillator strengths cause the plasmon, the spectrum simulated at SAOP/TZ2P level is made of many low-intensity peaks well scattered on all the 3.5-4.5 eV range. Additional spectra calculated with the lower quality basis sets DZ and TZP are given in Supplementary material ${ }^{59}$. While the DZ basis set was found not to be suitable, the TZP basis set leads to spectrum very similar to that obtained with the TZ2P basis set. Using the potential model LB94, the spectrum is found to be sensitive to the core pseudopotential. Using the small core Ag.3d/TZ2P 
version (the inner core orbitals were kept frozen up to the $3 \mathrm{~d}$ shell while $4 \mathrm{~s}, 4 \mathrm{p}, 4 \mathrm{~d}$, and $5 \mathrm{~s}$ electrons are in the valence), two bands are obtained at 3.86 and $4.20 \mathrm{eV}$, while the use of the large core Ag.4p/TZ2P (the $4 \mathrm{~s}$ and $4 \mathrm{p}$ electrons are treated as core electrons and only $4 \mathrm{~d}$ and $5 \mathrm{~s}$ electrons are explicitly considered), leads to a single band consisted of six main peaks in the 4.15-4.4 eV range. Of course, increasing the fwhm (0.08 eV in Figure 4) in the Gaussian broadening would make the more similar curves, except that the plasmon would be blueshifted by $0.3 \mathrm{eV}$ using $\mathrm{Ag} .4 \mathrm{p}$ core with respect to the use of $\mathrm{Ag}$.3d. We have also calculated the absorption spectrum with the BP86 GGA-type density functional and the two different schemes of frozen core. Using both Ag.3d and Ag.4p frozen cores, the spectrum is characterized by a strong density of states, with many low-intensity peaks scattered on all the range of energy starting from about $3 \mathrm{eV}$. However, the use of the large core Ag.4p leads to remove transitions below $3.5 \mathrm{eV}$. As expected the LB94 potential model gives more satisfactory spectra that BP86 does. However, it is not easy to conclude about the use of large versus small frozen core. The use of a frozen core is mandatory to take into account the scalar relativistic effects at low computational cost. The use of large-core pseudopotentials is questionable due to the significant radial overlap of the 5 s orbital with the inner $4 \mathrm{~d}$ shell, and that of the $4 \mathrm{~d}$ with $4 \mathrm{p}$ and $4 \mathrm{~s}$ ones. But using a small frozen core requires to be able to calculate correctly the additional correlation and exchange interactions. In the present case, the difference between the two LB94 spectra is a greater spacing between the dominant peaks when the small frozen core Ag.3d scheme is used. That can give rise to a break down of the band into two smaller bands if the curve of the absorption spectrum is drawn with a small fwhm in the Gaussian broadening process. It is well known that the LB94 potential model has deficiencies in the inner molecular regions $37,38,70$, and then it may be better to use a large frozen core as it was made in previous works concerning the optical response of noble metal nanoclusters ${ }^{19,33}$, keeping in mind that it could cause a blueshift of about 0.3 $\mathrm{eV}$.

The percentage of the $d$ character in the transitions calculated at LC-M06L and CAMB3LYP levels is weak, less than $10 \%$ (see Table I), and then the plasmon band is mainly due to contributions from $s \rightarrow s p$ intraband transitions. In contrast, the percentage of the $d$ character is calculated in the range of 50-90\% at BP86, SAOP and LB94 levels. At BP86 level, the main transition at $3.59 \mathrm{eV}$ presents a $d$ character of about $40 \%$. At SAOP level, the dominant band centered at $3.68 \mathrm{eV}$ presents a $d$ character of about $65 \%$. Finally, with 
LB94 the percentage is about $60 \%$ for the main band when using the Ag.4p frozen core, and $90 \%$ and $65 \%$ for the first and second bands at 3.86 and $4.20 \mathrm{eV}$ respectively when the small Ag.3d frozen core is used. Therefore, we can conclude that the plasmon band is mainly associated to contributions from $d \rightarrow s p$ interband transitions when GGA-type functionals or potential models are used. Although the AC potentiel models (LB94 and SAOP) give spectra somewhat similar to those obtained using LC functionals, the description of the plasmon differs. The $\Lambda$ value calculated from equation (3) is 0.6-0.7 for $s \rightarrow s p$ intraband transitions, and 0.35 for $d \rightarrow s p$ interband transitions. These $4 d \rightarrow 5 s 5 p$ transitions present a long-range charge-transfer character because of a relatively low overlap between occupied $d$-type orbitals, quite localised on atoms, and virtual orbitals distributed on an outer region, and the significant blue shift induced by the Hartree-Fock exchange. They are expected to be properly described with a correct asymptotic potential including HF exchange at long range. For $\mathrm{Ag}_{55}^{+}$, the asymptotic behavior as $-1 / r$ at long range seems not to be sufficient to well describe the optical response, an amount of Hartree-Fock exchange seems required.

Interestingly, we have used the equation (2) by distinguishing contributions from electrons of the 13-atom core and those from the 42-atom outer shell, and by extending it to occupied orbitals in the excited states (unoccupied states in the ground state). At CAM-B3LYP level, the contribution to the plasmon-like band of the electrons of the 13 -atom core is $67 \%$ (and $33 \%$ for electrons of 42 -atom outer shell), while the excited orbitals reached during the transition are mainly situated on the outer shell (61\%).

\section{Absorption spectra of $\mathrm{Ag}_{55}^{3-}$}

We have considered the clusters $\mathrm{Ag}_{55}^{3-}$ because it corresponds to a electronic shell closing in the $I_{h}$ symmetry. Absorption spectra calculated at LC-M06L, CAM-B3LYP, SAOP and LB94 levels are given in Figure 5. Additional spectra with several basis sets of lower quality are given in Supplemental materials ${ }^{59}$. In particular, our calculations using LB94 and SAOP associated with the DZ basis set, as used in a previous work ${ }^{33}$, leads to significant changes in the spectrum, i.e. a blueshift and a decrease in the number of peaks. At both SAOP/TZ2P and LB94/T2ZP levels, the spectrum of $\mathrm{Ag}_{55}^{3-}$ is somewhat similar to that of $\mathrm{Ag}_{55}^{+}$, the main difference being a small redshift of about $0.05-0.1 \mathrm{eV}$. The redshift is much more significant with LC-M06L and CAM-B3LYP functionals since the main transition is located at 3.82 and 

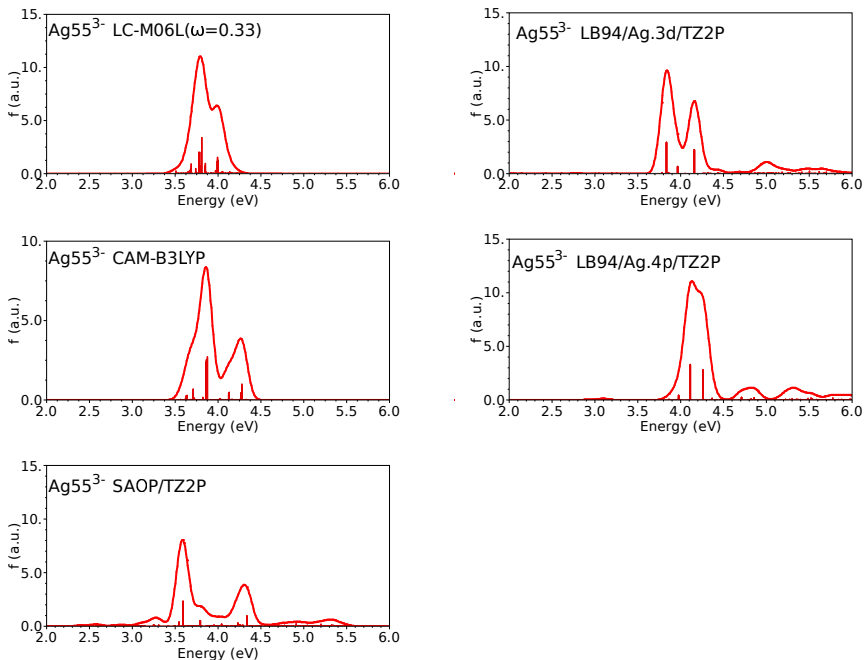

FIG. 5. Absorption spectra of $\mathrm{Ag}_{55}^{3-}$.

$3.86 \mathrm{eV}$ at LC-M06L and CAM-B3LYP respectively for $\mathrm{Ag}_{55}^{3-}$ compared to 4.20 and $4.14 \mathrm{eV}$ for $\mathrm{Ag}_{55}^{+}$. Similarly to $\mathrm{Ag}_{55}^{+}$, the excitations are mainly associated to $s$-type electrons when using LC-M06L and CAM-B3LYP, while the contribution of $d$-type electrons are much more significant in SAOP and LB94 simulations.

\section{CONCLUSION}

We have presented the absorption spectra of $\mathrm{Ag}_{20}$ and $\operatorname{Ag}_{55}^{q}(q=+1,-3)$ nanoclusters in the framework of the time-dependent density functional theory (TDDFT) using several families of density functionals. We have shown that the plasmon-like band of $\mathrm{Ag}_{20}$ is due to transitions implying $s$ electrons when either a range-separated hybrid (RSH) density functional including an amount of Hartree-Fock exchange at long range, namely LC-M06L, LC-BP86, LC- $\omega$ PBE, CAM-B3LYP and $\omega$ B97x, or an asymptotically corrected (AC) model potentials, namely LB94, SAOP and BP-GRAC-LB, is used. In contrast, for $\mathrm{Ag}_{55}^{q}, q=-1$ or +3 , the description of the plasmon differs with the scheme used (RSH versus AC) though the calculated spectra are somewhat similar. Using RSH methods, the plasmon-like band is again associated to excitations of $s$ electrons, with a very few contribution of $d$ electrons. But when using $\mathrm{AC}$ model potentials, the plasmon is associated to transitions implying mainly $d$-type electrons. Using semi-local exchange functionals (GGAs or meta-GGAs) leads to a red-shift of about $0.5 \mathrm{eV}$ of the plasmon band and an overestimation of the role of $d$ electrons 
in the plasmon-like band.

Our observations suggest that the $4 d \rightarrow 5 s 5 p$ transitions has some Rydberg character in the case of $\mathrm{Ag}_{20}$. But in the case of $\mathrm{Ag}_{55}^{q}$, where the $\mathrm{AC}$ model potentials fail to correct the deficiencies of GGAs, but the RSH functionals are able to provide a more accurate description, the $d \rightarrow s p$ interband transitions present a long-range charge-transfer character with a relatively low overlap between occupied and virtual orbitals and thus a significant blueshift is obtained when an portion of Hartree-Fock exchange is included at long range. The $d \rightarrow s p$ interband transitions are properly described with a correct asymptotic potential.

Our analysis of absorption spectra of $\mathrm{Ag}_{55}$ shows that an amount of non-local exchange at long-range in the framework of the adiabatic linear-response formulation of TDDFT is required to give a description of the plasmon-like band consistent with the well established classical interpretation. However the AC model potentials give somewhat similar spectra, at much low cost, though they may overestimate the role of $d$-type electrons in the excitations. Our results show that the description of the plasmon-like band in the TDDFT approach depends on the functional used even if the exact position of the plasmon is relatively easy to reproduce. An experimental evidence of the $s p$ or $d$ character of the electronic transitions would be necessary to obtain the correct description and validate the choose of the functional.

Present conclusions are expected to be transferable to larger nanoclusters. The description of the plasmon band in the language of TDDFT, and using RSH functionals, as a sum of few peaks associated to excitations of $s$ valence electrons seems to be consistent with the classical interpretation as a collective excitation of valence $s$-electrons.

\section{ACKNOWLEDGMENTS}

The author thanks AR Allouche for helpfull discussions. The author thanks the Pôle Scientifique de Modélisation Numérique (PSMN) at Lyon, France, and the GENCIIDRIS(Grant i2013086864) center for generous allocation of computational time.

\section{REFERENCES}

${ }^{1}$ D. D. Evanoff Jr. and G. Chumanov, ChemPhysChem 6, 1221-1231 (2005).

${ }^{2}$ S. M. Morton, D. W. Silverstein, and L. Jensen, Chem. Rev. 111, 3962-3994 (2011). 
${ }^{3}$ V. Bonačić-Koutecký, V. Veyret, and R. Mitrić, J. Chem. Phys. 115, 10450-10460 (2001).

${ }^{4}$ M. Harb, F. Rabilloud, and D. Simon, Chem. Phys. Lett. 476, 186-190 (2009).

${ }^{5}$ S. Lecoultre, A. Rydlo, J. Buttet, C. Felix, S. Gilb, and W. Harbich, J. Chem. Phys. 134, 074302 (2011).

${ }^{6}$ S. Lecoultre, A. Rydlo, J. Buttet, C. Felix, S. Gilb, and W. Harbich, J. Chem. Phys. 134, 074303 (2011).

${ }^{7}$ S. Lecoultre, A. Rydlo, J. Buttet, C. Felix, S. Gilb, and W. Harbich, J. Chem. Phys. 134, 184504 (2011).

${ }^{8}$ S. Fedrigo, W. Harbich, and J. Buttet, Int. J. Mod. Phys. B 06, 3767-3771 (1992).

${ }^{9}$ J. Tiggesbumker, L. Kller, H. O. Lutz, and K. H. Meiwes-Broer, Chem. Phys. Lett. 190, 42 (1992).

${ }^{10}$ J. Tiggesbumker, L. Kller, K. H. Meiwes-Broer, and A. Liebsch, Phys. Rev. A 48, R1789 (1993).

${ }^{11}$ S. Fedrigo, W. Harbich, and J. Buttet, Phys. Rev. B 47, 10706-15 (1993).

${ }^{12}$ M. Harb, F. Rabilloud, and D. Simon, Chem. Phys. Lett. 449, 38-43 (2007).

${ }^{13}$ M. Harb, F. Rabilloud, D. Simon, A. Rydlo, S. Lecoultre, F. Conus, V. Rodrigues, and C. Felix, J. Chem. Phys. 129, 194108 (2008).

${ }^{14}$ K. Baisha, J. C. Idrobo, S. Ogut, M. Yang, K. Jackson, and J. Jullinek, Phys. Rev. B 78, 075439 (2008).

${ }^{15}$ C. M. Aikens, S. Li, and G. C. Schatz, J. Phys. Chem. C 112, 11272-11279 (2008).

${ }^{16}$ M. Harb, F. Rabilloud, and D. Simon, J. Chem. Phys. 131, 174302 (2009).

${ }^{17}$ M. Harb, F. Rabilloud, and D. Simon, Phys. Chem. Chem. Phys. 12, 4246-4254 (2010).

${ }^{18}$ H. C. Weissker and C. Mottet, Phys. Rev. B 84, 165443 (2011).

${ }^{19}$ N. Durante, A. Fortunelli, M. Broyer, and M. Stener, J. Phys. Chem. C 115, 6277-6282 (2011).

${ }^{20}$ F. Rabilloud, Eur. Phys. J. D 67, 18 (2013).

${ }^{21}$ H. W. X.L. Lozano, C. Mottet, J. Phys. Chem. C 117, 3062-3068 (2013).

${ }^{22}$ M. L. Tiago, J. C. Idrobo, S. Ogut, J. Jellinek, and J. R. Chelikowsky, Phys. Rev. B 79, 155419 (2009).

${ }^{23}$ F. Rabilloud, J. Phys. Chem. A 117, 4267-4278 (2013).

${ }^{24}$ V. V. Kresin, Phys. Rev. B 51, 1844 (1995).

${ }^{25}$ L. Serra and A. rubio, Phys. Rev. Lett. 78, 1428 (1997). 
${ }^{26}$ J. Lermé, Eur. Phys. J. D 10, 265 (2000).

${ }^{27}$ M. Gaudry, J. Lermé, E. Cottancin, M. Pellarin, J. L. Vialle, M. Broyer, B. Prével, M. Treilleux, and P. Mélinon, Phys. Rev. B 64, 085407 (2001).

${ }^{28}$ E. Runge and E. K. U. Gross, Phys. Rev. Lett. 52, 997 (1984).

${ }^{29}$ R. van Leeuwen, Int. J. Mod. Phys. B 15, 1969 (2001).

${ }^{30}$ M. E. Casida, in Recent Advances in Density Functional Methods. Part I, p. 155 (D.P. Chong Ed. (Singapore, World Scientific), 1995).

${ }^{31}$ J. C. Idrobo and S. T. Pantelides, Phys. Rev. B 82, 085420 (2010).

${ }^{32}$ G. Barcaro, M. Broyer, N. Durante, A. Fortunelli, and M. Stener, J. Phys. Chem. C 115, 24085-24091 (2011).

${ }^{33}$ G. T. Bae and C. M. Aikens, J. Phys. Chem. C 116, 10356-10367 (2012).

${ }^{34}$ J. P. Perdew, R. G. Parr, M. Levy, and J. L. B. Jr., Phys. Rev. Lett. 49, 1691 (1982).

${ }^{35}$ D. J. Tozer and N. C. Handy, J. Chem. Phys. 108, 2545 (1998).

${ }^{36}$ R. van Leeuwen and E. J. Baerends, Phys. Rev. A 49, 2421 (1994).

${ }^{37}$ P. R. T. Schipper, O. V. Gritsenko, S. J. A. van Gisbergen, and E. J. Baerends, J. Chem. Phys. 112, 1344 (2000).

${ }^{38}$ M. Gruning, O. V. Gritsenko, S. J. A. van Gisbergen, and E. J. Baerends, J. Chem. Phys. 114, 652 (2001).

${ }^{39}$ M. J. G. Peach, P. Benfield, T. Helgaker, and D. J. Tozer, J. Chem. Phys. 128, 044118 (2008).

${ }^{40}$ A. Dreuw and M. Head-Gordon, Chem. Rev. 105, 4009-4037 (2005).

${ }^{41}$ T. Leininger, H. Stoll, H. J. Werner, and A. Savin, Chem. Phys. Lett. 275, 151-160 (1997).

${ }^{42}$ B. Anak, M. Bencharif, and F. Rabilloud, RSC Adv. 4, 13001-13011 (2014).

${ }^{43}$ A. D. Becke, Phys. Rev. A 38, 3098 (1988).

${ }^{44}$ J. P. Perdew, Phys. Rev. B 33, 8822 (1986).

${ }^{45}$ J. P. Perdew, K. Burke, and M. Ernzerhof, Phys. Rev. Lett. 77, 3865-3868 (1996).

${ }^{46}$ C. Lee, W. Yang, and R. G. Parr, Phys. Rev. B 37, 785-789 (1988).

${ }^{47}$ J. P. Perdew, J. A. Chevary, S. H. Vosko, K. A. Jackson, M. R. Pederson, D. J. Singh, and C. Fiolhais, Phys. Rev. B 46, 6671 (1992).

${ }^{48}$ Y. Zhao and D. G. Truhlar, J. Chem. Phys. 125, 194101 (2006). 
49. M. Tao, J. P. Perdew, V. N. Staroverov, and G. E. Scuseria, Phys. Rev. Lett. 91, 146401 (2003).

${ }^{50}$ J.-W. Song, T. Hirosawa, T. Tsuneda, and K. Hirao, J. Chem. Phys. 126, 154105 (2007).

${ }^{51}$ A. D. Becke, J. Chem. Phys. 98, 5648-5652 (1993).

${ }^{52}$ Y. Zhao and D. G. Truhlar, Theor. Chem. Account 120, 215-241 (2008).

${ }^{53}$ T. Yanai, D. P. Tew, and N. C. Handy, Chem. Phys. Lett. 393, 51-57 (2004).

${ }^{54}$ J.-D. Chai and M. Head-Gordon, J. Chem. Phys. 128, 084106 (2008).

${ }^{55}$ O. A. Vydrov, J. Heyd, A. V. Krukau, and G. E. Scuseria, J. Chem. Phys. 125, 074106 (2006).

${ }^{56}$ M. J. Frisch, G. W. Trucks, H. B. Schlegel, G. E. Scuseria, M. A. Robb, J. R. Cheeseman, G. Scalmani, V. Barone, B. Mennucci, G. A. Petersson, H. Nakatsuji, M. Caricato, X. Li, H. P. Hratchian, A. F. Izmaylov, J. Bloino, G. Zheng, J. L. Sonnenberg, M. Hada, M. Ehara, K. Toyota, R. Fukuda, J. Hasegawa, M. Ishida, T. Nakajima, Y. Honda, O. Kitao, H. Nakai, T. Vreven, J. A. Montgomery, Jr., J. E. Peralta, F. Ogliaro, M. Bearpark, J. J. Heyd, E. Brothers, K. N. Kudin, V. N. Staroverov, R. Kobayashi, J. Normand, K. Raghavachari, A. Rendell, J. C. Burant, S. S. Iyengar, J. Tomasi, M. Cossi, N. Rega, J. M. Millam, M. Klene, J. E. Knox, J. B. Cross, V. Bakken, C. Adamo, J. Jaramillo, R. Gomperts, R. E. Stratmann, O. Yazyev, A. J. Austin, R. Cammi, C. Pomelli, J. W. Ochterski, R. L. Martin, K. Morokuma, V. G. Zakrzewski, G. A. Voth, P. Salvador, J. J. Dannenberg, S. Dapprich, A. D. Daniels, A. Farkas, J. B. Foresman, J. V. Ortiz, J. Cioslowski, and D. J. Fox, "Gaussian 09 Revision A.1," Gaussian Inc. Wallingford CT 2009 .

${ }^{57}$ D. Andrae, U. Haussermann, M. Dolg, H. Stoll, and H. Preuss, Theor. Chim. Acta 77, 123-141 (1990).

${ }^{58}$ P. J. Hay and W. R. Wadt, J. Chem. Phys. 82, 299 (1985).

${ }^{59}$ See supplemental material at [URL will be inserted by AIP] for seven figures that show the geometrical structure of $\mathrm{Ag}_{20}$ and $\mathrm{Ag}_{55}$ (Figure $\mathrm{S}-1$ ), the absorption spectra calculated with several density functionals, RECP and basis set (Figures S-2, S-3, S-5, S-6, S-7), the isosurface of the electron density difference between the excited and ground states for some of the main peaks of $\mathrm{Ag}_{20}$ clusters (Figure S-4).

${ }^{60}$ D. Figgen, G. Rauhunt, M. Dolg, and H. Stoll, Chem. Phys. 311, 227-244 (2005).

${ }^{61}$ F. Weigend and R. Ahlrichs, Phys. Chem. Chem. Phys. 7, 3297-3305 (2005). 
${ }^{62}$ iK. A. Peterson and C. Puzzarini, Theo. Chem. Acc. 114, 283-296 (2005).

${ }^{63}$ G. te Velde, F. Bickelhaupt, E. Baerends, C. F. Guerra, S. van Gisbergen, J. Snijders, and T. Ziegler, J. Comput. Chem. 22, 931-967 (2001).

${ }^{64}$ E. van Lenthe, E. Baerends, and J. Snijders, J. Chem. Phys. 101, 9783 (1994).

${ }^{65}$ E. van Lenthe, J. Snijders, and E. Baerends, J. Chem. Phys. 105, 6505 (1996).

${ }^{66}$ A. R. Allouche, J. Comput. Chem. 32, 174-182 (2011).

${ }^{67}$ H. Haberland, Nature 494, E1-E2 (2013).

${ }^{68}$ L. Jensen, L. L. Zhao, and G. C. Schatz, J. Phys. Chem. C 111, 4756-4764 (2007).

${ }^{69}$ E. Loginov, L. F. Gomez, N. Chiang, A. Halder, N. Guggemos, V. V. Kresin, and A. F. Vilesov, Phys. Rev. Lett. 106, 233401 (2011).

${ }^{70}$ W. Cencek and K. Szalewicz, J. Chem. Phys. 139, 024104 (2013). 
Description of plasmon-like band in silver clusters: the importance of the long-range Hartree-Fock exchange in time-dependent density-functional theory simulations

Franck Rabilloud ${ }^{1}$

Institut Lumière Matière, UMR5306 Université Lyon 1 - CNRS,

Université de Lyon 69622 Villeurbanne Cedex, France

(Dated: 11 September 2014)

\section{Supplemental Materials}

The supporting information includes the geometrical structure of $\mathrm{Ag}_{20}$ and $\mathrm{Ag}_{55}$ (Figure S-1), the absorption spectra calculated with several density functionals, RECP and basis sets (Figures S-2, S-3, S-5, S-6, S-7), the isosurface of the electron density difference between the excited and ground states for some of the main peaks of $\mathrm{Ag}_{20}$ clusters (Figure $\mathrm{S}-4$ ). 

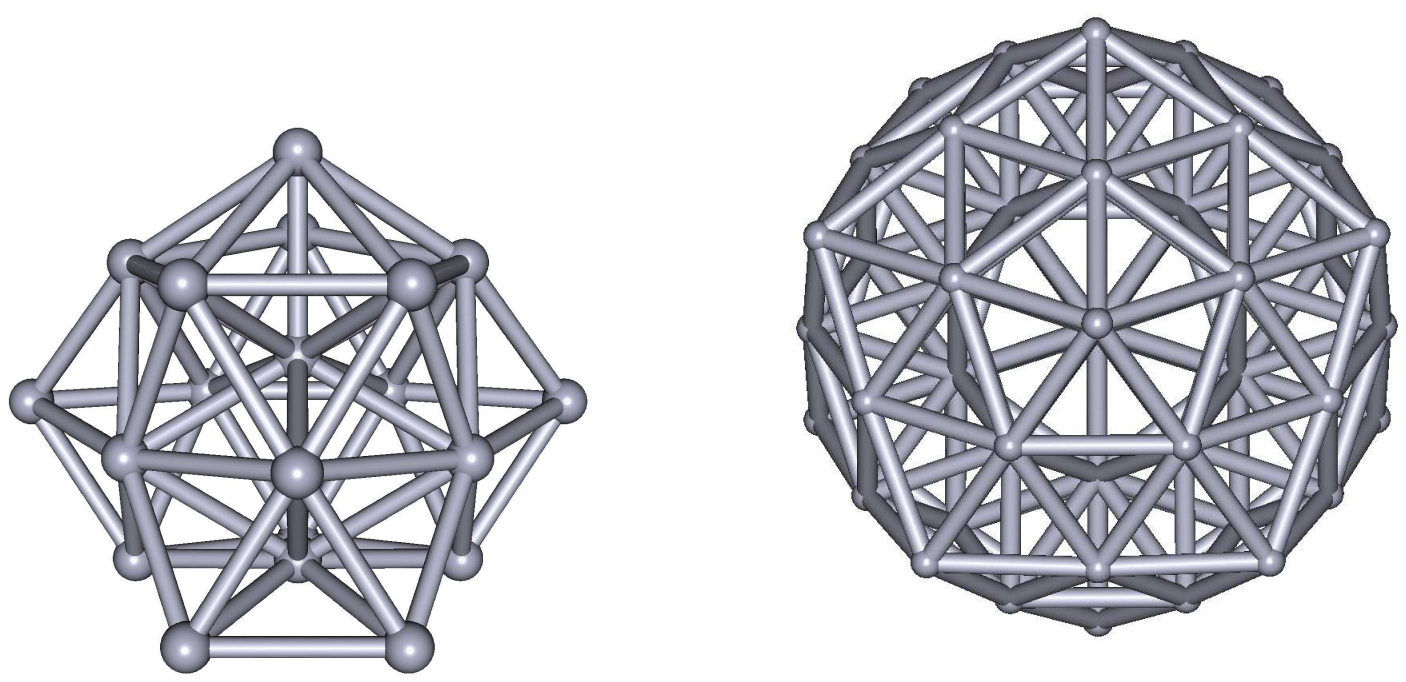

FIG. S-1. Supplemental Material: Structures of $\mathrm{Ag}_{20}$ and $\mathrm{Ag}_{55} \cdot \mathrm{Ag}_{20}$ is the lowest-energy isomer $\left(C_{s}\right.$-symmetry) taken from our previous study with BP86 calculations (M. Harb et al, J. Chem. Phys. 129, 194108 (2008)), it consists of a core formed by a 13-atom icosahedron. $\mathrm{Ag}_{55}$ is the icosahedral structure ( $I_{h}$ symmetry). 

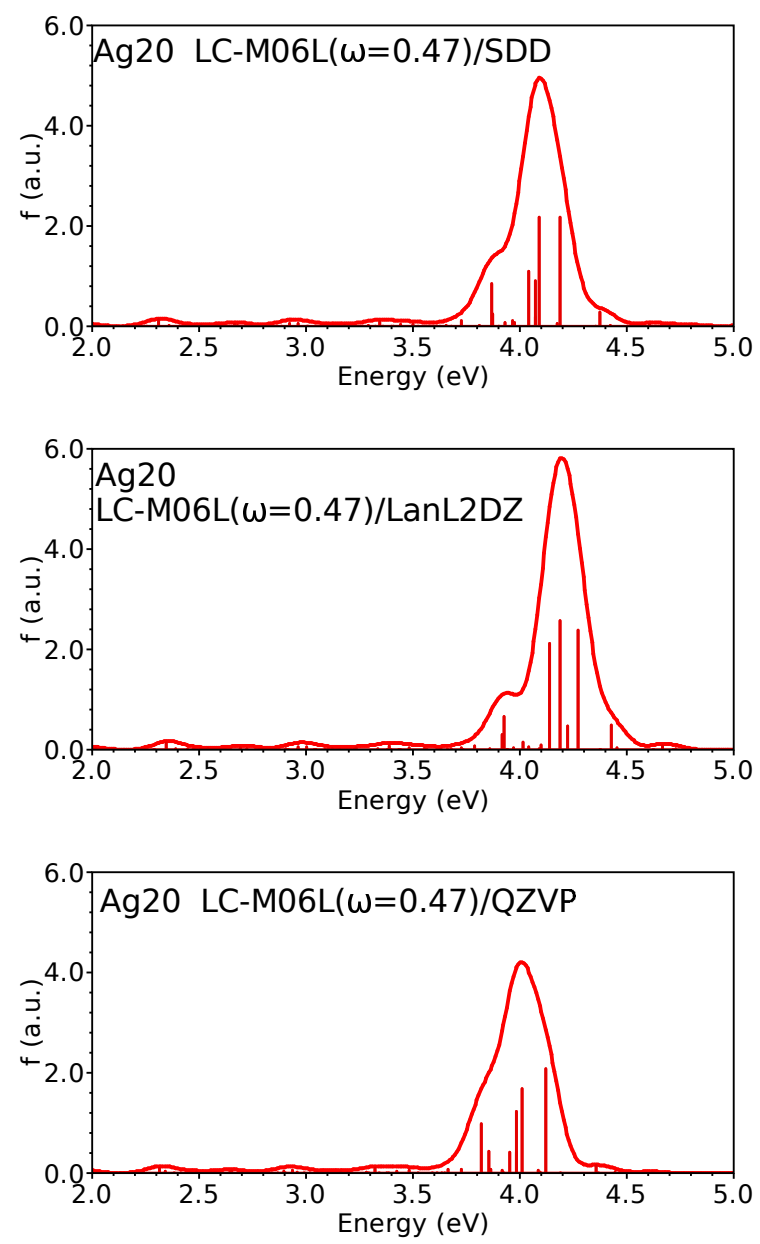

FIG. S-2. Supplemental Material: Absorption spectra of $\mathrm{Ag}_{20}$ using three basis sets and effective core pseudopotentials, namely SSD (8s7p6d contracted into 6s5p3d, D. Andrae et al, Theor. Chem. Acta. 77, 123-141 (1990)), LanL2DZ (7s6p4d contracted into 3s3p2d, Hay and Wadt, J. Chem. Phys. 82, 299 (1985)), and QZVP (10s8p7d3f1g contracted into 7s5p4d3f1g, Weigend and Ahlrichs, Phys. Chem. Chem. Phys. 7, 3297-3305 (2005)). 

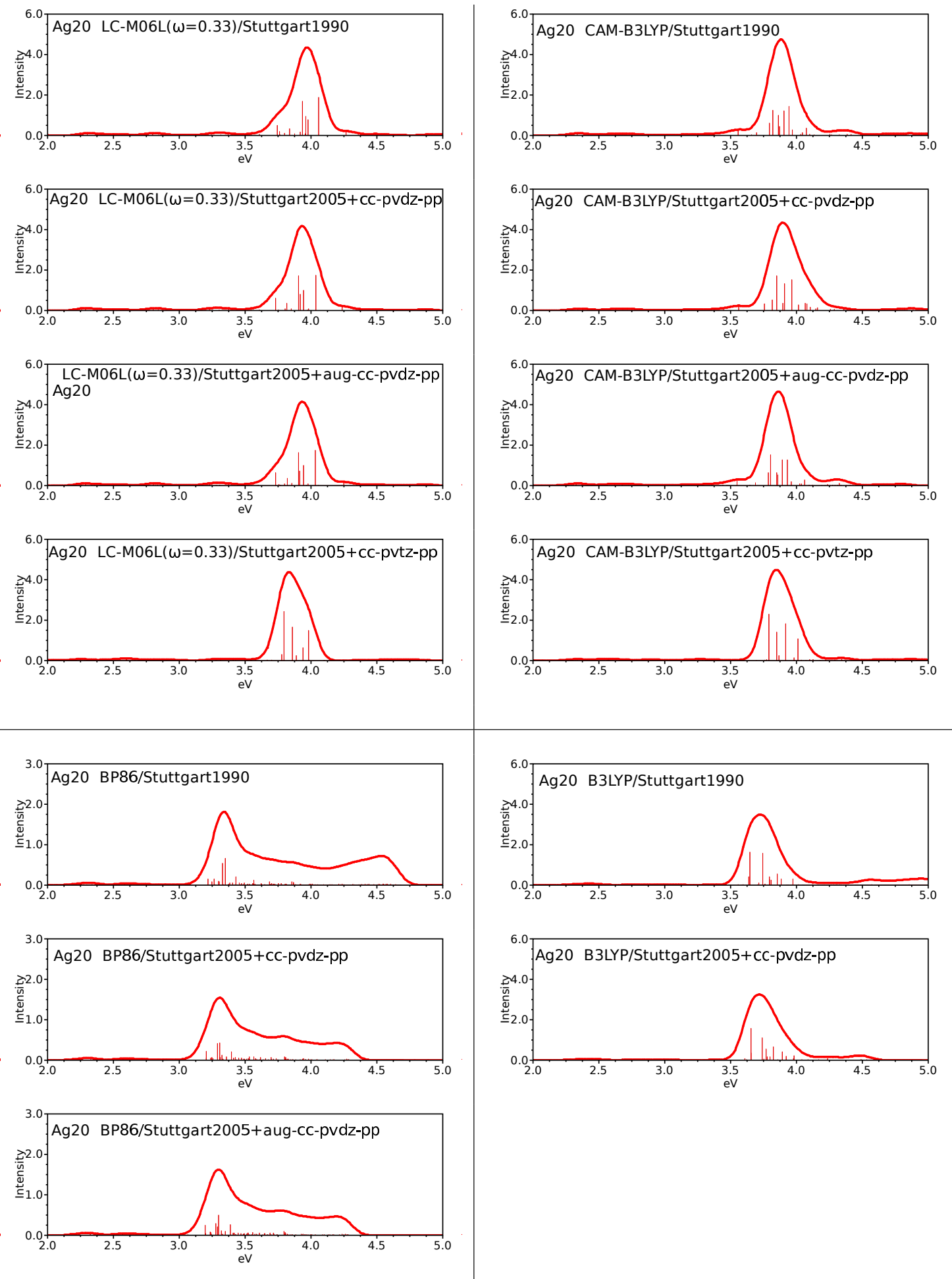

FIG. S-3. Supplemental Materials: Absorption spectra of $\mathrm{Ag}_{20}$ calculated using several density functionals, namely B3LYP, BP86, CAM-B3LYP, LC-M06L( $\omega=0.33)$, and several effective core pseudopotentials, Stuttgart1990 (also called SDD in Fig.S-2) [D. Andrae, U. Haussermann, M. Dolg, H. Stoll, H. Preuss, Theor. Chim. Acta 77, 123-141 (1990)] and Stuttgart2005 [D. Figgen, G. Rauhut, M. Dolg, H. Stoll, Chem. Phys. 311, 227-244 (2005)] with several associated basis sets [K. A. Peterson, C. Puzzarini, Thoer. Chem. Acta 114 283-296 (2005)]. cc-pvdz-pp is a doubly zeta valence + polarization quality basis set, aug-cc-pvdz-pp is a diffuse function-augmented version of cc-pvdz-pp, cc-pvtz-pp is a triple zeta valence + polarization quality basis set. 


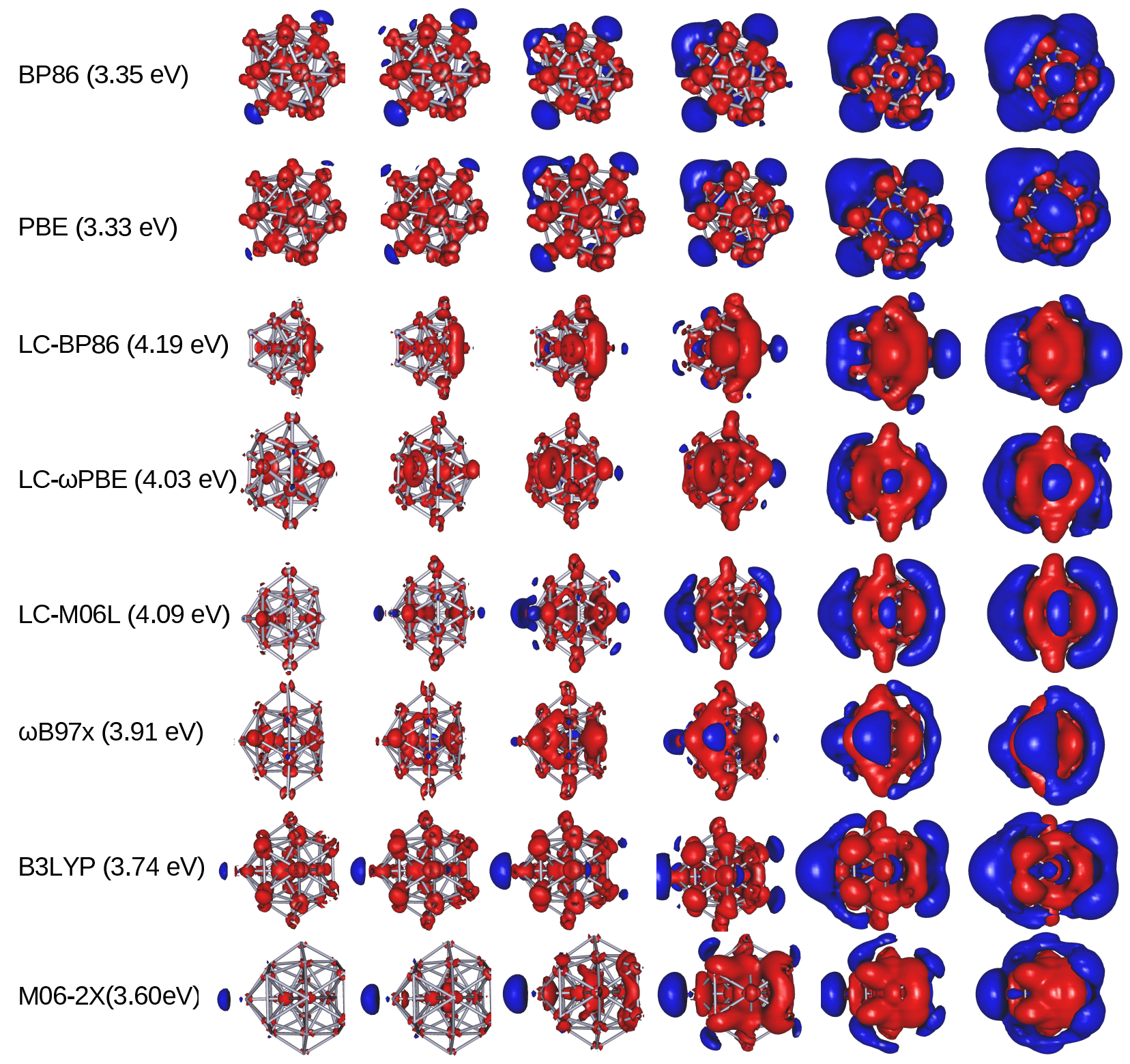

FIG. S-4. Supplemental Material: Isosurface of the electron density difference between the excited and ground states for some of the main peaks of $\mathrm{Ag}_{20}$ clusters. The isovalues are 0.0005, 0.0004, 0.0003, 0.0002, 0.0001 and 0.00005 a.u. respectively. Red colored regions correspond to the depletion of the electron density during the transition while the blue regions correspond to the accumulation of electrons. 

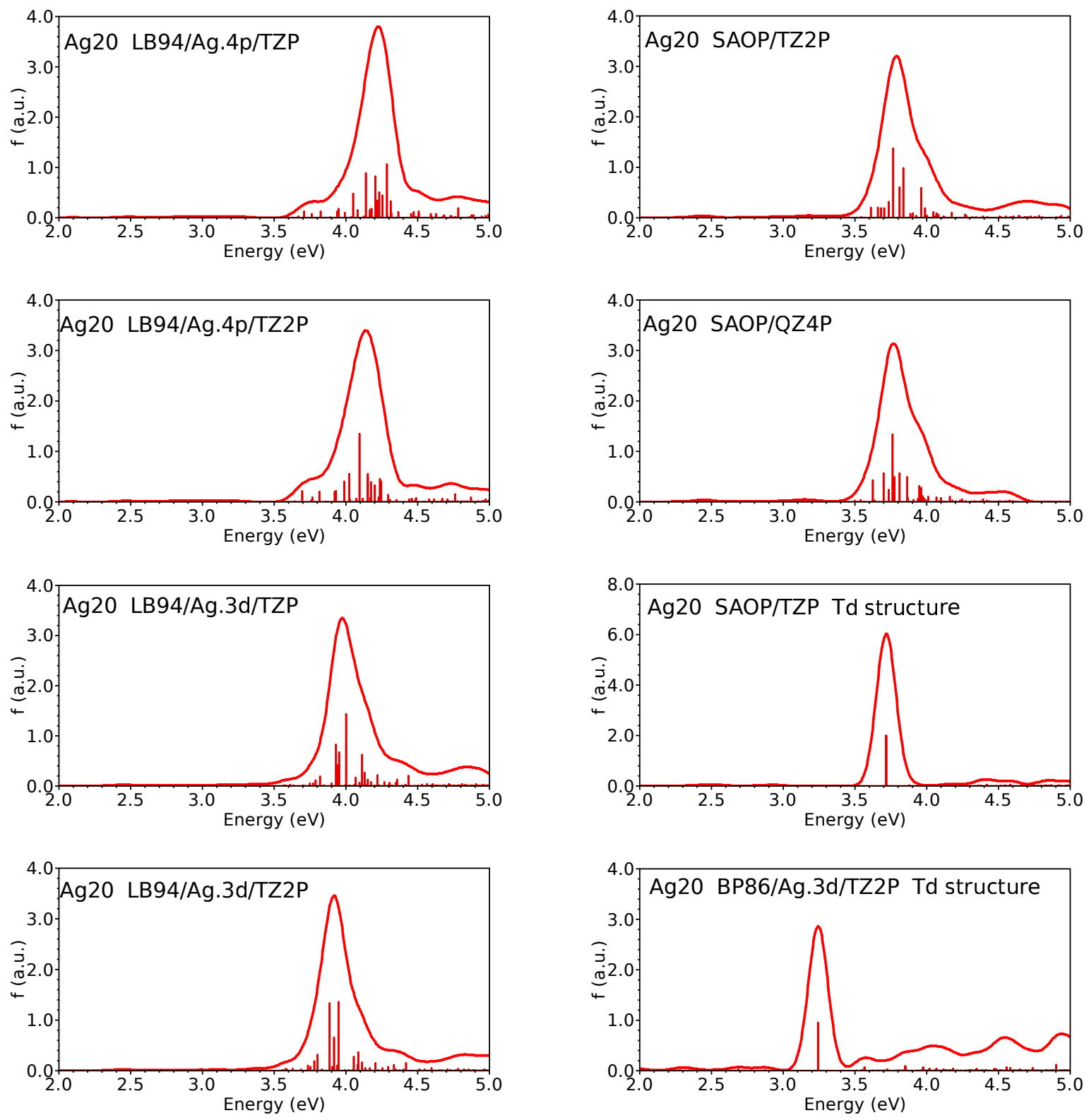

FIG. S-5. Supplemental Material: Additional absorption spectra of $\mathrm{Ag}_{20}$. 

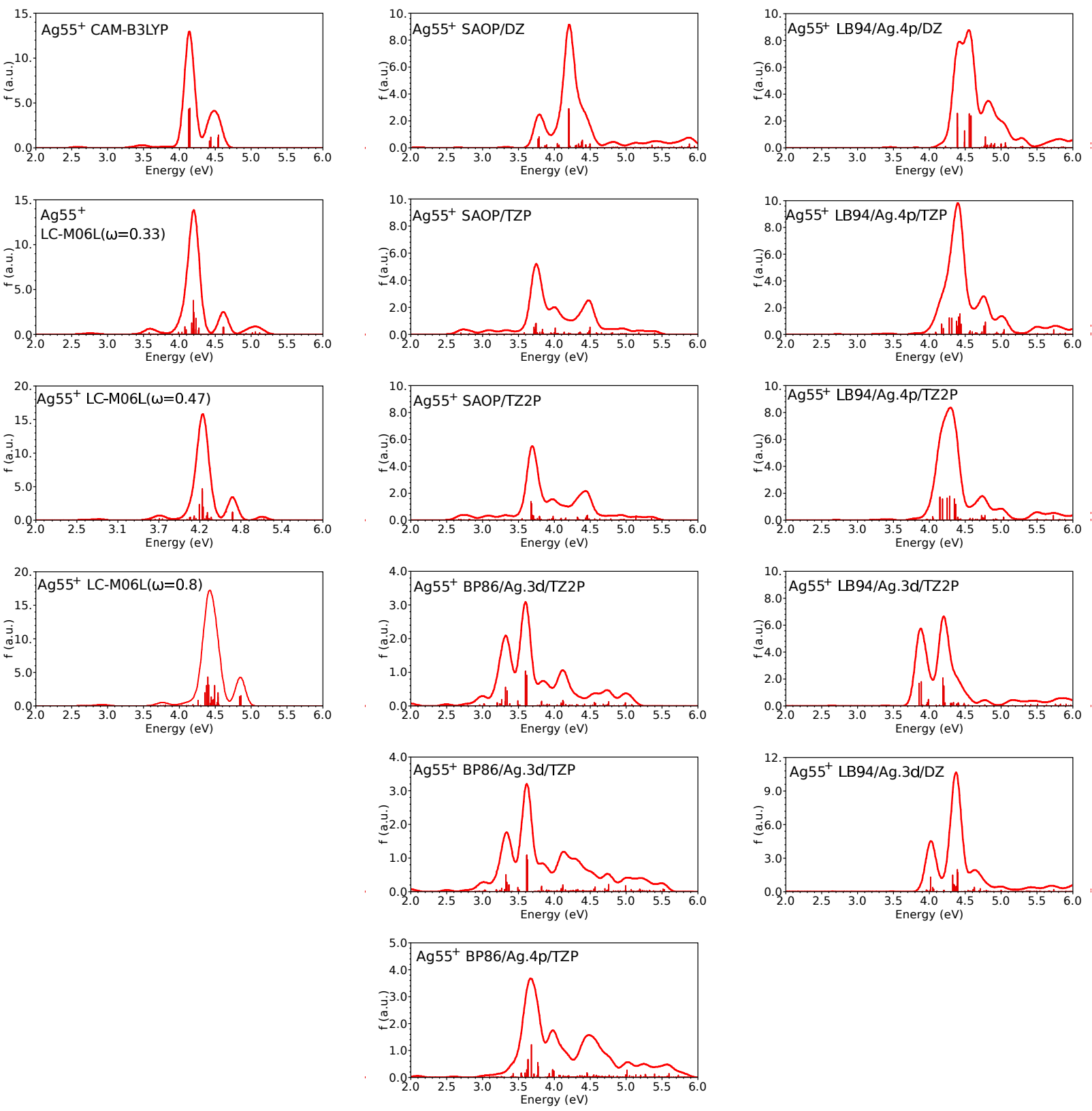

FIG. S-6. Supplemental Material: Additional absorption spectra of $\mathrm{Ag}_{55}^{+}$. 

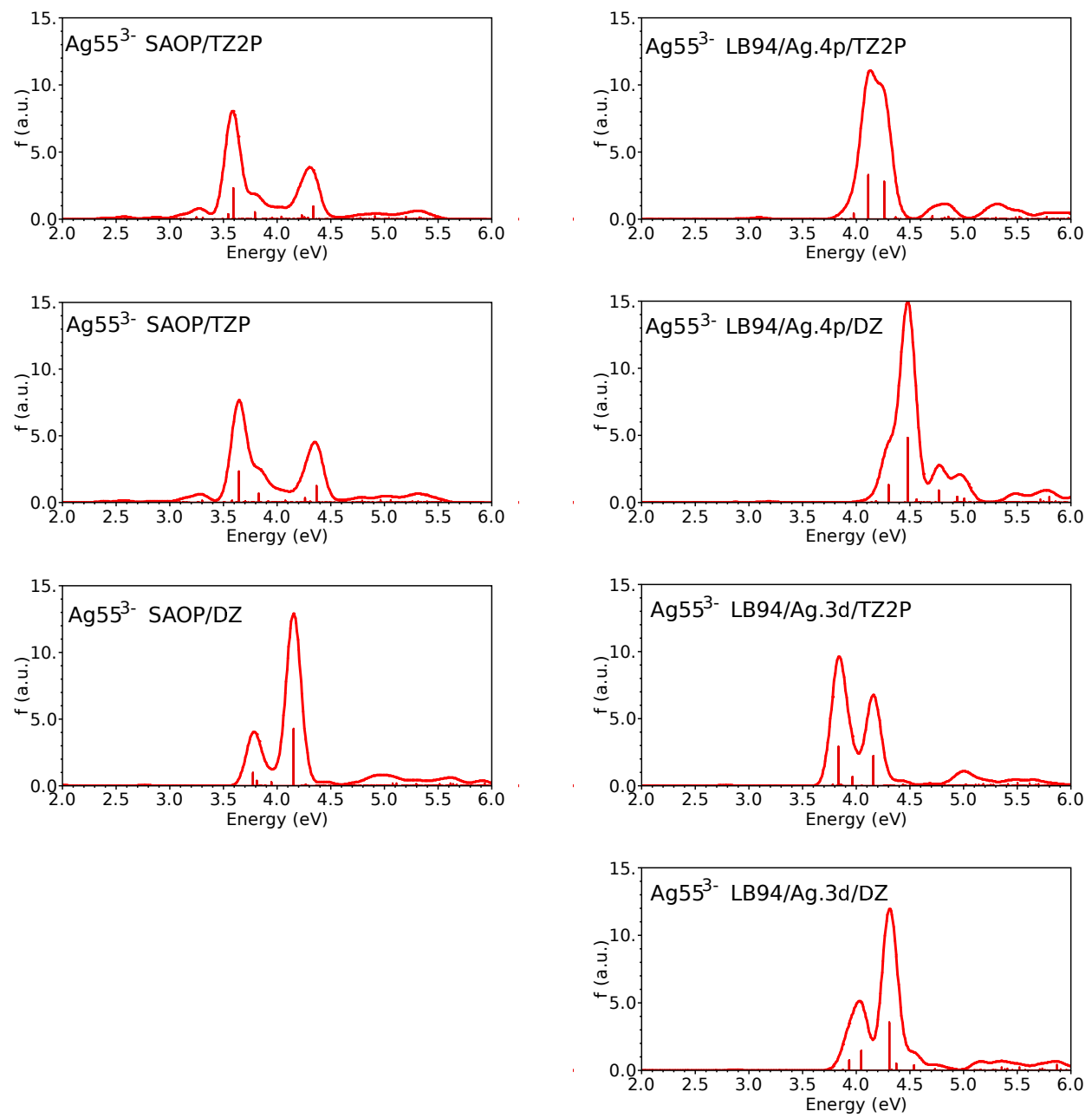

FIG. S-7. Supplemental Material: Additional absorption spectra of $\mathrm{Ag}_{55}^{3-}$ at SAOP and LB94 levels using several basis sets. 